\title{
Kritische Theorie, Systemtheorie und der Treibsand der Soziologie ${ }^{1}$
}

\section{Helmut Staubmann}

Zusammenfassung: In der Soziologiegeschichte gilt die Tradition systemtheoretischer Soziologie, wie sie im Gefolge von Talcott Parsons entwickelt wurde, als eine affirmative Theorie, deren Unzulänglichkeiten von verschiedenen Strömungen kritischer Theorie aufgedeckt und korrigiert wurden. Der Aufsatz zeigt die Einseitigkeit dieser Interpretation. Ein Grundmotiv sowohl der Kritischen Theorie als auch der systemtheoretischen Soziologie ist der Versuch einer Syntheseleistung unterschiedlicher geistesgeschichtlicher Strömungen und Teildisziplinen der Humanwissenschaften. Sie mündeten zwar in unterschiedlichen Versionen von Gesellschafts- und Handlungstheorien, gemeinsam ist ihnen aber eine Opposition zur gegenwärtig vorherrschenden Fragmentierung und empiristischen Ausrichtung soziologischer Praxis. Am Beispiel des Lebenswerkes von Harold J. Bershady wird sowohl die Verbindung eines kritischen Anspruches mit systemtheoretischer Handlungstheorie deutlich als auch die Bedeutung eines einheitlichen theoretischen Bezugsrahmens und die Notwendigkeit von dessen kritischer Weiterentwicklung.

Schlüsselwörter: Handlungstheorie, Kritische Theorie, Marxismus, Systemtheorie, Wissenschaftstheorie der Sozialwissenschaften

1 Der Beitrag basiert auf einer bearbeiteten Übersetzung meines Besprechungsessays: Staubmann, Helmut. 2015. Marx, Parsons, and the Quicksands of History. Harold J. Bershady's Intellectual and Personal Life Journey. European Journal of Cultural and Political Sociology 2: 56-61

H. Staubmann $\bowtie$

Universität Innsbruck, Institut für Soziologie

Universitätsstraße 15, 6020 Innsbruck, Österreich

E-Mail: helmut.staubmann@uibk.ac.at 


\title{
Critical Theory, Systems Theory and the Quicksands of Sociology
}

\begin{abstract}
In the common historiography of sociology, the tradition of systems-theoretical sociology as it was developed by and in the wake of Talcott Parsons is characterized as an affirmative ideology, the shortcomings of which were uncovered and corrected by various currents of critical thought. The essay demonstrates the one-sidedness of this interpretation. A basic motif, both, of critical theory as well as of systems theory, is the attempt at reaching a synthesis of different currents in the history of thought and of subdisciplines of the humanities. Although they resulted in different versions of action theory and theories of society they share a common opposition to the contemporary dominating fragmentation of sociological praxis. At the example of the life work of Harold J. Bershady, the linking of critique and action theory becomes evident as well as the meaning of a unified theoretical frame of reference and the necessity of its critical further elaboration.
\end{abstract}

Keywords: Action Theory, Critical Theory, Marxism, Philosophy of the Social Sciences, Systems-Theory

Theorie der Gesellschaft oder Sozialtechnologie, so der Titel eines von Jürgen Habermas und Niklas Luhmann gemeinsam verfassten Buches (1971) scheint im Common Sense der soziologischen Geschichtsschreibung die disjunkte Alternative von kritischer und systemtheoretischer Orientierung auf den Punkt zu bringen. Luhmann wird dabei von seinem Kontrahenten die unkritische funktionale Beschreibung der Gesellschaft und damit die Legitimation bestehender gesellschaftlicher Verhältnisse attestiert. Mit der Fortführung des Funktionalismus seines frühen Lehrers Talcott Parsons hat Luhmann auch das Erbe einer Kritik getragen, die sich zunächst einmal an dessen Werk entzündet hatte. Die wichtigste Quelle zeitgenössischer Erkenntnis, Wikipedia, stellt das Parsonssche Werk, wenn man so will, entsprechend defizitär dar: es könne weder Konflikte noch sozialen Wandel erfassen und sei auch sonst das Gegenstück progressiver intellektueller Strömungen. Dass eine Theorie mit einem solchen Label nicht mehr nachgefragt wird, ist wohl nicht verwunderlich. „Who now reads Parsons?“ hatte Jeffrey Alexander einen Vortrag zum Status des Funktionalismus begonnen in Anspielung an eine Formulierung Parsons' zu Herbert Spencer in der Einleitung zu The Structure of Social Action (1937).

Wenn man den zeitgenössischen Theoriediskus betrachtet, so scheint das Schicksal, das das Parsonssche Werk ereilte, aber auch seine ehemaligen Kontrahenten, jedenfalls einige sehr prominente, ereilt zu haben. Um auf die berühmte Kontroverse zwischen Luhmann und Habermas zurückzukommen, so ist die Präsenz beider Werke in der Soziologie stark zurückgegangen. Die zentrale historische Achse der kritischen Theorie, nämlich Adorno und Habermas, spielt in der gegenwärtigen Soziologie nur mehr eine untergeordnete Rolle. Einige Konzepte von Pierre Bourdieu und Michel Foucault und vor allem empirische Forschung auf Basis von „grounded 
theory“ sind in die Lücke nachgerückt. Die Bruchstelle scheint nicht mehr affirmativ versus kritisch sondern „grand theory“ versus ad-hoc Forschung. War letztere noch in der Tradition der klassischen kritischen Theorie als affirmativ verpönt, so ist diese nun in eine Allianz mit neueren Formen von Gesellschaftskritik eingetreten.

Die Dynamik dieser Entwicklung möchte ich in der Folge am Beispiel des Lebenswerkes von Harold J. Bershady darlegen² ${ }^{2}$ Bershady zählt zum engeren Kreis von Parsons-Schülern. Er hat sich vor allem mit wissenssoziologischen Fragen und Fragen der Ideologie befasst. 2014 ist seine Autobiographie erschienen, die seine eigene intellektuelle Entwicklung im Kontext der gesellschaftlichen Ereignisse nachzeichnet. When Marx Mattered, so der Titel der Autobiographie, stellt die gängige Darstellung des quasi natürlichen Gegensatzes von gesellschaftskritischem Anspruch und systemtheoretisch-funktionaler Theorie in Frage.

Die historischen Fakten zur Entwicklung der Parsonsschen Theorie des Handelns und der Rolle Parsons' in den soziopolitischen Kernthemen des 20. Jahrhunderts sowie seiner Schülerinnen widersprechen auch völlig dem vorherrschenden Bild. Parsons hatte sich in wissenschaftlichen Arbeiten und in seinen praktischen politischen Aktivitäten gegen die totalitären Strömungen in Europa gestellt, insbesondere gegen den Nationalsozialismus (s. Gerhardt hrsg. 1992) und hatte sich für den Wiederaufbau eines demokratischen Europas eingesetzt. Er half vertriebenen Europäern in den USA Fuß zu fassen, unter anderem Theodor W. Adorno und sah in der Wiedereröffnung des Frankfurter Institutes für Sozialforschung unter Leitung von Max Horkheimer eine wichtige Maßnahme zur intellektuellen Konsolidierung in Deutschland (vgl. Parsons 1953). Entsprechend lud ihn Horkheimer auch nach Frankfurt und zur Mitarbeit am Journal für Sozialforschung ein (vgl. Horkheimer 1953). Diese Aktivitäten waren für Parsons eine Art „Marshall-Plan des Geistes“ (s. Staubmann und Tschernitz 2016). Auch das Salzburg Seminar in American Studies zählte dazu, an dem Parsons in den Nachkriegsjahren dreimal als Gastprofessor tätig war und dort unter anderem den jungen Ralf Dahrendorf unterrichtete ${ }^{3}$. Innenpolitisch unterstützte Parsons den Kampf um die Rechte der Schwarzen in den USA und war in der McCarthy Ära mit Anschuldigungen kommunistischer Agitation konfrontiert. Viele bedeutende SozialwissenschafterInnen, deren Arbeiten heute als Gegenposition zu Parsonsschen Handlungstheorie angesehen werden, hatten von ihm wichtige Anregungen erhalten. So schrieb Clifford Geertz 1974 in einem Brief freimütig an seinen Lehrer: „I have learned more from you

2 Erwähnt sei auch, dass Harold Bershady mein „Sponsor“, wie es im Amerikanischen heißt, in meiner Zeit (199697) als Visiting Scholar an der University of Pennsylvania war. Es haben sich daraus eine Reihe von Kooperationen ergeben. Unter anderem war er 1999 Teilnehmer der Konferenz Talcott Parsons - Current Relevance of a Theory Program in Innsbruck (siehe auch seinen mit Victor M. Lidz verfassten Beitrag in der daraus entstanden Publikation Action Theory; siehe Methodological Studies (Staubmann, hrsg. 2006).

3 Dahrendorf wurde dann sehr früh zu einem Kritiker Parsons“ und entwickelte die Identität seiner „Konflikttheorie“ aus der Kontrastierung zu Parsons (siehe Dahrendorf 1955 und 1957). 
than from anyone I have ever studied with, and no one has done more to set the general frame of my thought". ${ }^{4}$

Ein Schüler Parsons', der eine wichtige Brücke zu Jürgen Habermas schlagen sollte, war Mark Gould. Bereits 1964, beim 15. Deutschen Soziologentag, der dem Werk Max Webers gewidmet war, hatte es eine Debatte zwischen Parsons und Habermas gegeben, bei der sich Parsons, wie er es in seinem Kommentar ausdrückte, von Habermas missverstanden fühlte (siehe die Dokumentation in Stammer hrsg. 1965). Gould, der sich auch mit kritischer Theorie im weiteren Sinne verbunden fühlte, sah es wohl als eine Aufgabe an Missverständnisse zwischen den beiden Theoriesträngen zu klären. Der ehemalige Assistent von Parsons verbrachte ein Forschungsjahr am Starnberger Max-Planck-Institut, das unter der Direktion von Jürgen Habermas stand. Deren Diskussionen motivierten Habermas zu einer intensiveren Auseinandersetzung mit Parsons' Theorie, was letztendlich in das Design der Theorie des kommunikativen Handelns (1981) einfloss. Gould lud Habermas zu einer Gastprofessur an sein Haverford College ein. Durch die Nähe zur University of Pennsylvania, Philadelphia, kam es zum Kontakt mit dem dortigen Parsons-Kreis und zu diesem zählte auch Harold J. Bershady.

\section{Die Synthese von Kritik und Systemtheorie bei Harold J. Bershady}

Der Marxismus, hat Jean Paul Sartre einmal gemeint, hatte ihn angezogen wie das Licht die Fliegen. Und, so fuhr Sartre fort, nachdem er alle Kategorien bürgerlichen Denkens abgestreift hatte, fühlte er sich plötzlich hilflos in einer neuen Situation, für die der Marxismus keinen Sinn mehr machte, weil er in einem Stillstand erstarrt war. Der Schimmer der Aufklärung, den Marx ausgestrahlt hatte und der sozialen Bewegungen in seinem Gefolge hatten in der Tat einen großen Teil der Intellektuellen des 20sten Jahrhunderts magisch angezogen, und, wie es die Sartresche Allegorie impliziert, ihnen an einem gewissen Punkt das Gefühl gegeben gebrannt zu werden von der Realität der Ereignisse, die sich unter dem Label Marxismus entwickelt hatten. Dieses von Sartre treffend gezeichnete Bild kam mir wieder in den Sinn, als ich die 2014 erschienenen Memoiren von Harold J. Bershady über seine intellektuelle Entwicklung und das ereignisreiche Leben, aus dem heraus er sich gebildet hatte, las. Und es gibt noch eine weitere verblüffende Analogie. Beide versuchen eine Synthese von zwei als unvereinbar geltenden intellektuellen Strömungen; in Sartres Fall waren es Existenzialismus und Marxismus, bei Bershady auch seine früh erfolgte Zuwendung zum Marxismus und seine spätere Begegnung mit dem Werk von Talcott Parsons.

Gnothi sauton - erkenne dich selbst! - die berühmte Inschrift auf dem Apollo Tempel in Delphi kann als das Leit-Postulat von Bershadys Lebensweg angesehen werden, das er in zeit-

4 Zur Bedeutung Parsons' für die Cultural Sociology siehe auch Staubmann 2016. 
gemäßer Weise als eine persönliche und soziologische Annäherung zum Wissen verfolgte. Es ist wohl relativ leicht einen Konsens zu erzielen, dass wir Wissen über uns selbst anstreben sollen, ein Wissen das untrennbar mit Wissen über unsere Gesellschaft und unsere Kultur verbunden ist. Das Enigma des Postulates liegt vielmehr in der Frage, wie das erreicht werden kann und, wenn überhaupt, wie wir gültiges Wissen von Täuschung unterscheiden können oder, etwas anders formuliert, richtiges von falschem Bewusstsein.

In seinem frühen Werk Ideology and Social Knowledge (1973, 2. Auflage 2014a) behandelt Bershady diese Fragen in einem rein theoretischen Sinne. Seine Autobiographie, die unter dem Titel When Marx Mattered (2014b) erschienen ist, platziert diese Frage im Kontext seiner persönlichen Geschichte. Die beiden Bücher sind gewissermaßen Zwillinge, die zusammengehören und sich gegenseitig ergänzen. Durch die Neuauflage von Ideology and Social Knowledge, das durch eine Einleitung erweitert ist, die die theoretischen Entwicklungen seit der Erstauflage 1973 zusammenfasst, sind beide Bände nun zugänglich.

Aber beginnen wir am Anfang. Die Vorgeschichte seiner intellektuellen Odyssee, wie es im Untertitel der Autobiographie heißt, führt zurück in einige entlegene Dörfer - Schtetls - in der Ukraine, wo seine jüdischen Eltern und andere Verwandte gelebt haben und, wegen des wachsenden Antisemitismus, letztlich das Land 1921 verließen. Seine Mutter erreichte als Erste die Neue Welt, sein Vater folgte nach einigen Stationen in Europa. Die Familie vereinigte sich wieder in Toronto und siedelte sich dort in einer Gegend mit jüdischen Einwanderern an. Das war der Ort, wo Harold 1929 auf die Welt kam. Sechs Jahre später übersiedelte die Familie, sehr zum Missfallen von Harold, nach Buffalo.

In den ersten Jahren wuchs er zweisprachig auf, Jiddisch und English. Die Mischung aus Hochkultur und Empfindsamkeit gegenüber sozialen und politischen Fragen, die sich aus seinem jüdischen Status ergaben, wird auf anschauliche Weise bereits für die ersten Lebensjahre in berührenden autobiographischen Geschichten und Anekdoten beschrieben - die Schwierigkeiten mit den ökonomisch engen Verhältnissen umzugehen. Der erfolgreiche Versuch ein Klavier zu kaufen und eine Klavierausbildung zu bekommen führte beinahe zu einer professionellen Pianisten-Karriere. Die Liebe zur Literatur und der sehr frühe Beginn einer Art philosophischer Selbst-Reflexion waren nicht zuletzt motiviert durch den Wunsch die schwierige Situation zu verstehen, mit Fragen des religiösen Glaubens zurande zu kommen und Antworten zu finden auf die politischen Fragen im Zusammenhang mit Antisemitismus und allgemeiner einer sozioökonomischen Umgebung, die als ungerecht empfunden wurde. Aus diesem frühen quasi philosophischen Keim erwuchs das spätere Interesse für Marxismus, das durch Verwandte bestärkt wurde, die an einem bestimmten Punkt offen ihre Verbundenheit zu marxistischen Ideen deklarierten.

Die Geschichte der frühen Jahre macht drei Kernfragen der Autobiographie verständlich, die Bershady umfassend zu beantworten versucht: 
1. Warum waren in den USA viele Marxisten und Sozialisten jüdische Amerikaner, wie er selbst, als er noch als Teenager Marxist wurde?

2. Welche waren die Einflüsse, die ihn und viele seiner marxistischen Freunde zu einer allgemeiner liberalen Anschauung leiteten, in manchen Fällen sogar zu einem „Gestalt-switch“ von einem linken Weltbild zum Konservativismus?

3. Welche Erfahrungen trugen dazu bei, dass er ein soziologischer Theoretiker wurde? Um sich diesen Fragen anzunähern wird der Leser/die Leserin durch die Wirrungen sozialer Stürme geführt um zu zeigen, wie der Autor versuchte mit diesen umzugehen und sie zu verstehen.

1946, einem Jahr nach Kriegsende, bewarb sich Bershady auf ein College und wurde von der University of Buffalo aufgenommen. Dort nahm er bald an einer Organisation mit dem Namen Youth for Democracy teil. Sie war ursprünglich von Veteranen gegründet worden und zog vor allem jüngere Studierende an. Eine Diskussionsgruppe über Marxismus, die später John Reed $C l u b$ genannt wurde, konsolidierte seine politische Orientierung. Eine Diskussion von Marx' Die deutsche Ideologie, ein Text, der später basal wurde für die Entstehung der Wissenssoziologie, brachte ihn schließlich dazu dieses Thema als Thema seiner Masterarbeit zu wählen. Der Club hatte Netzwerke etabliert, die ihn in Kontakt mit der Kommunistischen Partei brachten, der er kurz vor seinem 18. Geburtstag beitrat. Nachdem er seinem Vater von diesem Schritt erzählte, erfuhr er von einer Episode, die einen symbolischen Charakter für seine späteren Erfahrungen bekommen sollte. Als junger Mann hatte sich sein Vater der Bolschewistischen Armee angeschlossen. Er selbst war kein Kommunist, setzte jedoch Hoffnungen in die kommunistische Bewegung für eine bessere zukünftige Gesellschaft und ein besseres Leben.

Sein Vater hatte immer wieder kleinere anti-semitische Vorfälle in der roten Armee erlebt. Dann kam es zu einer besonders erniedrigenden Erfahrung mit Offizieren, aus der er den Schluss zog, dass deren Führer genauso ungerecht und mit Vorurteilen behaftet waren, wie sie es vor der Revolution waren. Diese Erfahrung veranlasste seinen Vater letztendlich das Land zu verlassen.

Wie im Falle seines Vaters desillusionierte auch den jungen Kommunisten bald die Kluft zwischen Theorie und Hoffnung einerseits und der Realität andererseits. Die Ereignisse, die man in der Sowjetunion beobachten konnte, waren weit weg vom Ideal einer friedlichen, offenen und gleichen Gesellschaft. Stattdessen, so schlussfolgerte Bershady, handelte es sich um einen Albtraum von Blut und Zerstörung. Über diese Erkenntnis legte er seine Mitgliedschaft in der Kommunistische Partei wieder zurück. Als ob es eines Beweises für die Richtigkeit seines Schrittes bedurft hätte, wurde er von einer Person im kommunistischen Hauptquartier erbracht, indem er mit Drohungen reagierte das FBI über die frühere Mitgliedschaft zu informieren.

Die Ernüchterung, die diese Erfahrungen bewirkten, zogen bei Bershady ein vertieftes Interesse an philosophischen Fragen nach sich, insbesondere zur Möglichkeit von „wirklichem“ Wissen im Gegensatz zu Täuschung und Ideologie. Ist jedes Wissen grundsätzlich an subjektive Perspektiven gebunden oder würde es möglich sein das „Gespenst des Relativismus“ 
(Bershady, S. 87) zu überwinden? Ein erstes Resultat war eine Prüfungsarbeit über Max Schelers Phänomenologie. Scheler sollte bis in Bershadys spätem Werk von Bedeutung bleiben, als er einen Band mit von ihm selbst übersetzen Aufsätzen unter dem Titel Max Scheler on Feeling, Knowing, and Valuing (1992) herausgab.

Nachdem er seine Masterarbeit über Karl Mannheims Wissenssoziologie fertiggestellt hatte, entschied sich Bershady für die University of Madison, Wisconsin um dort ein Doktoratsstudium zu absolvieren, das er 1959 begann. Der Hauptgrund für diese Entscheidung war der Soziologie-Professor Hans H. Gerth, ein Immigrant aus Deutschland, der dort ein Student bei Karl Mannheim gewesen war und später einige von Max Webers Aufsätzen ins Englische übersetzte und zwar mit seinem Studenten C. Wright Mills. Nebenbei bemerkt, war Gerth ein MitStudent von Talcott Parsons in einem Karl Jaspers Seminar in Heidelberg gewesen.

Bershady kam erstmalig in einem Theoriekurs mit dem Werk von Talcott Parsons in Kontakt, bei dem Auszüge aus The Structure of Social Action behandelt wurden. Es stellte sich heraus, dass dies zu einer folgenreichen Begegnung werden sollte. Ganz gegen die vorherrschenden Urteile der linken intellektuellen Gefährten, nahm er Parsons' Handlungstheorie als ein ambitioniertes „Syntheseschema“ (S. 126) wahr, ähnlich der Bestrebungen von Karl Marx nach einer umfassenden Theorie gesellschaftlicher Dynamik. Nach Bershadys Verständnis war Parsons auf Augenhöhe mit Marx. Es kam ihm das Verdienst zu, die soziale Theorie grundlegend weiterentwickelt zu haben, indem er ein Jahrhundert wissenschaftlicher Neuerungen einarbeitete. Für Bershady resultierte daraus eine unvoreingenommene Interpretation von Parsons' Theorie, während er noch an Fragen marxistischer Debatten interessiert blieb.

Zusammen mit seinem Lehrer Hans Gerth versuchte er Georg Lukacs' marxistische Wissenssoziologie, nämlich das Werk Geschichte und Klassenbewusstsein, zu übersetzen. Zu Bershadys intellektuellem Hauptanliegen wurde aber Talcott Parsons, über den er sich entschloss eine Dissertation zu verfassen mit dem Titel The Apriori in Talcott Parsons'Social Theory. Aus dieser Arbeit ging nach grundlegenden Überarbeitungen das Buch Ideology and Social Knowledge hervor.

Das Jahr 1962 eröffnet ein neues biographisches Kapitel, indem er mit seiner Frau und deren neugeborenem Sohn nach Philadelphia zog um an der University of Pennsylvania als „Instructor“ zu arbeiten. Die University of Pennsylvania hatte weithin die Reputation eine jüdische Universität zu sein. In einem intellektuell anregenden Soziologie-Institut sollte er wichtige Beziehungen zu KollegInnen entwickeln, zuerst mit E. Digby Baltzell und Renée C. Fox und später mit Victor M. Lidz. Es war, wie es Bershady ausdrückte, „the Age of Aquarius“, das Zeitalter der Gegenkultur mit der Herausbildung eines neuen Lebensstils und der Neuen Linken mit einem neuen Zugang zum Marxismus. Die civil rights movement mit Martin Luther King Jr. erreichte einen Höhepunkt in den 60er Jahren und Bershady war dort in Washington, DC am 28. August 1963 als die berühmte Ansprache „I have a dream..." gehalten wurde. 
In den frühen 70er Jahren verbrachte Bershady ein Sabbatical in Europa, was ihm die Zeit gab die Arbeit an Ideology and Social Knowledge zu einem Abschluss zu bringen. Bershady wurde damit in der Folge zu einem assoziierten Professor gehoben.

1974 fand dann das erste persönliche Treffen mit Talcott Parsons statt. Parsons war 1973 von seiner Professur in Harvard emeritiert und wurde von Renée Fox, zu dieser Zeit Vorstand des Institutes für Soziologie, zu einer Gastprofessur an die University of Pennsylvania, Philadelphia eingeladen, die mit dem Wintersemester 1974 begann. Bald folgten Arbeitsgespräche über das sogenannte „Human Condition Paradigm“ gemeinsam mit Willy De Craemer, Renée Fox und Victor Lidz. Diese Gruppe wurde gelegentlich von Robert Bellah, A. Hunter Dupree und Clifford Geertz verstärkt.

Bershadys erstes Zusammentreffen mit Talcott Parsons begann mit einer bemerkenswerten kleinen Anekdote. Bershady stand in einem überfüllten Büro, in dem eine rege Betriebsamkeit von SekretärInnen, Studierenden und KollegInnen herrschte. Bershady wartete auf das Eintreffen von Talcott Parsons. Nach einiger Zeit fragte er Victor Lidz, der gerade hereingekommen war, ob Parsons überhaupt schon angekommen sei. Lidz lachte und sagte, ja, er steht direkt hinter dir und zeigte auf einen Mann, der in einer der aufliegenden Kursbeschreibungen blätterte. Der Mann war um einiges älter, glatzköpfig, rundlich, klein. Seine Tweed-Jacke war etwas verknittert und seine Krawatte war unscheinbar und hängte etwas schief. Das war Talcott Parsons?!? Aufgrund der Bedeutung und der Berühmtheit von Talcott Parsons hatte Bershady eine eindrucksvolle Gestalt erwartet und entdeckte eine etwas scheu wirkende Person. Im Zuge vieler professioneller und privater Zusammenkünfte sollte Parsons für ihn später zu einer Art von ,intellektuellem Vater“" (S. 214) werden. Ihre Beziehung fand ein viel zu frühes jähes Ende. Im Frühjahr 1979 reiste Parsons nach Deutschland um den 50. Jahrestag der Verleihung seines Doktorates an der Universität Heidelberg zu feiern (siehe die Dokumentation in Schluchter hrsg. 1979). Nach diesem Festakt fuhr Parsons auf Einladung von Horst Helle nach München um am Institut für Soziologie einige Vorträge zu halten. Dort erkrankte er und verstarb am 8. Mai. „Wir haben die Nachricht innerhalb von wenigen Stunden erhalten. Es war erschütternd“ (S. 215).

Für Bershady war kein „Gestalt-switch“ notwendig um sich nach seiner frühen Begeisterung für marxistische Ideen nunmehr Parsons' allgemeiner Theorie des Handelns zuzuwenden. Ein unvoreingenommener Zugang in beiden Fällen erlaubte eine differenzierte Beurteilung ihres theoretischen Gehaltes und dessen, was er in beiden Fällen als Mängel bewertete. Nach Bershadys Beobachtung wurde Marx zur Zeit der Gegenkultur und der Studentenbewegung nicht tiefgreifend rezipiert. Er wurde einfach eher mit Protest assoziiert, als dass man sich mit ihm intellektuell auseinandersetzte. In Bezug auf Parsons kann diese Beobachtung einfach umgekehrt werden. Wie im Falle von Marx wurden auch die Werke von Parsons wenig gelesen und ohne gute Gründe eher mit Konservativismus assoziiert als mit dem, was er theoretisch wirklich ge- 
leistet hatte. Parsons war zum „black beast of the radicals” (S. 183) geworden, wie es Bershady treffend formuliert. Bershady war weit davon entfernt sich solchen Klischees hinzugeben und versuchte in einer Verbindung von theoretischen Fragen und Antworten beider Traditionen zu einer neuen Synthese zu gelangen.

Das Buch Ideology and Social Knowledge kann als ausgezeichnetes Beispiel dafür angesehen werden. Bershady machte es sich darin zur Aufgabe, die großen Errungenschaften von Parsons herauszuarbeiten und gleichzeitig deren Grenzen aufzuzeigen. „Das Buch war eine Art langer Brief an Parsons“ (S. 182-3) erinnert sich Bershady. Parsons antworte darauf höflich aber irgendwie irritiert in einem ausführlichen „Antwortschreiben“ - einer Rezension, die ursprünglich im damaligen „Parsons-Journal“ Sociological Inquiry publiziert wurde. Bershady antwortete wiederum darauf und Parsons publizierte sowohl seine Rezension als auch Bershadys Antwort in seinem Sammelband Social Systems and the Evolution of Action Theory (1977).

Bershady führt in Ideology and Social Knowledge den Leser/die Leserin auf eine Reise durch Parsons' Theorieentwicklungen und den verschiedenen Versionen seiner hochkomplexen theoretischen Strukturen, die er, im Gegensatz zu vielen anderen KommentatorInnen, in einer kohärenten Logik verbunden sah. Die Komplexität des Bezugsrahmens des Handelns ist seiner Meinung notwendig um einen naiven Empirismus und Reduktionismus in seinen verschiedenen Spielarten zu vermeiden. In When Marx Mattered findet sich eine Stelle, die den Geist des Parsonsschen Unterfangens einfach und doch treffend auf den Punkt bringt: “... human life is ... made up of many things, each one distinct yet actively influencing and providing vital energies to all the others, and to ignore the contributions of any one is to warp our understanding of them all." (S. 59). Parsons' begriffliche Differenzierungen, notwendig um den vielen Dingen, aus denen das menschliche Leben besteht, gerecht zu werden, ist eine großartige Leistung. Wissenschaft versucht aber eine Antwort auf kausale Fragestellungen. Es ist gerade diese Frage der kausalen Analyse, von Notwendigkeiten und hinreichenden Bedingungen, bei der Bershady die Unzulänglichkeiten der Parsonsschen Theorie diagnostiziert. Dies beginnt mit der Frage der kausalen Interpretation von Beziehungen zwischen den Subsystemen. Was bedeutet etwa die vermeintliche Notwendigkeit unserer körperlichen Bedingungen für die Muster sozialer Interaktion? Diese Typen von kausalen Beziehungen sind für Bershady nicht hinlänglich ausgearbeitet. Er vermutet die Annahme des sogenannten Hempel-Modells oder des mechanistischen Newtonschen Modells für kausale Erklärungen, die für Handlungsanalysen aber unangemessen erscheinen. Anstelle des Versuches der Formulierung kausaler Gesetze auf Grundlage abstrakter Eigenschaften von Objekten müsste sich eine adäquate Erklärungsform, die sich in Übereinstimmung mit dem kategorialen Schema des Bezugsrahmens des Handelns befindet, auf interpretative Methoden stützen. Bershady plädiert für die Erweiterung der Parsonsschen Konvergenzmethode, indem die Handlungstheorie mit Phänomenologie, den Arbeiten von Alfred Schütz, Wilhelm Dilthey, Georg Simmel und insbesondere Max Scheler konfrontiert 
wird. Kurz, Bershady sieht eine Art positivistischen Restbestand in Parsons' Bezugsrahmen des Handelns. Dieser könnte ihm zufolge überwunden werden durch eine stärkere Orientierung an phänomenologischen Traditionen in den Sozial- und Kulturwissenschaften. ${ }^{5}$

Parsons zeigte sich in seiner Antwort nicht völlig überzeugt, fühlte sich offensichtlich einigermaßen missverstanden und reagierte, indem er sich in der Tradition der Evolutionstheorie (dem Mendelschen Modell, wie er es nannte) verortete, weiters in der Tradition von Whiteheads Prozessphilosophie und sich gleichzeitig von den Traditionen mechanistischer Methodologie distanzierte. Es mögen wohl Missverständnisse zu einzelnen Fragen auf beiden Seiten eine Rolle gespielt haben. Die Leistung von Bershadys Buch besteht jedoch darin, die Aufmerksamkeit auf methodologische Kernaspekte der Handlungstheorie gerichtet zu haben, die noch nicht vollständig gelöst sind und die Richtung für die weitere Arbeit gewiesen zu haben.

Die Theorie des Handelns ist, wie Bershady bekräftigt, weit davon entfernt abgeschlossen zu sein. Sie wird eine offene Theorie bleiben, offen für weiteren Fortschritt im Geiste dessen, was am Anfang die Konvergenzthese genannt wurde.

Soziologie und andere Disziplinen der Humanwissenschaften haben nach Parsons aufs Große und Ganze die Arbeit an einem einheitlichen Bezugsrahmen ausgeblendet. Soziologische Forschung wurde seit Parsons, wie Bershady in beiden seiner Bücher beobachtet, eine Art von

5 Max Preglau hat auf meinen Vortrag zum Thema „Soziale Kausalität“ im Rahmen eines Institutskolloquiums im Sommersemester 2016 einen schriftlichen Kommentar verfasst, der sich weitgehend mit diesen Positionen von Harold J. Bershady deckt. Hier ein Auszug aus seinen verblüffend ähnlichen Argumenten: „Wie schon in der Diskussion betont, stimme ich in vielen Punkten mit Dir überein: - mit der Kritik eines theorielosen Empirismus, der Korrelationen ex post kausal interpretiert; - mit der Kritik eines theorielosen Herangehens an die soziale Realität, das sich dabei auf Grounded Theory beruft... - mit der Ablehnung lern- bzw. verhaltenstheoretischer Reiz-Reaktionsmodelle; - mit der Kritik an deterministischen Theorien der Abhängigkeit eines Systems von seiner Umwelt. Im Positiven teile ich die Ansicht, dass Soziologie theoriegeleitet vorgehen soll, sowie auch die Idee, der „Eigengesetzlichkeit" des Sozialen. ...

Nicht nachvollziehen kann ich die von Dir vertretene strikte Disjunktion von Lebenswelt und Wissenschaft und die Polemik gegen das „Sinnverstehen“ a la Max Weber, und zwar aus folgendem Grund: Mit der kultursoziologischhandlungstheoretischen Tradition der Soziologie gehe ich davon aus, dass die Eigenlogik des Sozialen zunächst einmal durch kulturelle Sinnzusammenhänge bestimmt ist und daher auch nur verstehend, und das heißt: auf Basis eines lebensweltlich fundierten Vorverständnisses (das der Interpret als sozialisiertes Kulturwesen mit dem zu interpretierenden Sinnzusammenhang verbindet), möglich. Genau das ist es was eine ethnografische Soziologie (und Anthropologie) und eine qualitative Sozialforschung tut und worauf auch quantitative Sozialforschung im Rahmen der explorativen Phase angewiesen ist. Einem Wesen vom Mars (oder von der Venus) das von allen lebensweltlichen Bezügen abgetrennt wäre, hätte mangels Sozialisation diese Möglichkeit gar nicht. Soziale Sinnzusammenhänge können freilich auch von den Intentionen und Vorstellungen ihrer Konstrukteure unabhängige Wirkungen und Bestandsvoraussetzungen haben, die sich durch eine bloß verstehende Rekonstruktion nicht erfassen lassen, sondern im Rahmen von lebensweltfernen, kontraintuitiven Theorien erklärt werden müssen. Hier scheint mir der rechtmäßige Platz für die von Dir vertretene soziologische Theorie funktionalistisch-systemtheoretischer Prägung zu sein (das ist natürlich eine von ... Habermas entlehnte Vorstellung). Aber selbst eine solche Theorie muss sich ihren Gegenstand, bevor sie nach dessen nichtreflektierten Voraussetzungen und nichtintendierten Effekten forscht, vorweg im Wege eines ethnographischen Verstehens erschließen. ..."(Preglau 2016). 
Flickwerk. Einige Konzepte von hier oder dort gelten als hinreichend um soziale Probleme zu behandeln oder um öffentliche Aufmerksamkeit zu erregen.

„Who remains troubled by the so-called 'quicksands of history'?", stellt Bershady die Frage mit Bezug zum Diskurs des 19. Jahrhunderts. Ich befürchte, dass das Hin- und Herschieben des Treibsandes, wie wir es mittlerweile seit einigen Jahrzehnten beobachten können, die großen Errungenschaften, den Humanwissenschaften eine solide begriffliche und methodologische Basis zu geben, verschütten. Die beiden Bücher Bershadys, das eine mit seinem bezaubernden Stil und bewegenden Geschichten, das andere mit seiner analytischen Brillanz, erinnern uns mit großer Deutlichkeit an die zur Verfügung stehenden Alternative.

\section{Literaturverzeichnis}

Bershady, Harold J. 1992. Max Scheler on Feeling, Knowing, and Valuing. Chicago: University of Chicago Press.

Bershady, Harold J. 2014a. Ideology and Social Knowledge. With a New Introduction by the Author. New Brunswick and London: Transaction Publishers (originally published in 1973 by Halsted Press, a division of John Wiley \& Sons, Inc.).

Bershady, Harold J. 2014b. When Marx Mattered. An Intellectual Odyssey. New Brunswick and London: Transaction Publishers.

Dahrendorf, Ralf. 1955. Struktur und Funktion. Talcott Parsons und die Entwicklung der soziologischen Theorie. Kölner Zeitschrift für Soziologie 4: 491-520.

Dahrendorf, Ralf. 1957. Soziale Klassen und Klassenkonflikt in der industriellen Gesellschaft. Stuttgart: Enke.

Geertz, Clifford. 1974. Letter to Talcott Parsons (April, 15). The Talcott Parsons Papers, Harvard University Archives.

Gerhardt, Uta 1993. Talcott Parsons on National Socialism. New York: Walter de Gruyter.

Habermas, Jürgen. 1981. Theorie des kommunikativen Handelns. 2 Bde. Frankfurt am Main: Suhrkamp Verlag.

Habermas, Jürgen und Niklas Luhmann. 1971. Theorie der Gesellschaft oder Sozialtechnologie. Frankfurt a. Main: Suhrkamp.

Horkheimer, Max. 1953. Letter to Talcott Parsons (November, 15). The Talcott Parsons Papers, Harvard University Archives.

Lidz, Victor, Bershady, Harold. 2006. Parsons' Tacit Metatheory. In: Action Theory. Methodological Studies, hrsg. Helmut Staubmann, 107-152. Berlin: LIT.

Parsons, Talcott. 1937. The Structure of Social Action. 2 Vols. New York: The Free Press.

Parsons, Talcott. 1953. Letter to Max Horkheimer (December, 15). The Talcott Parsons Papers, Harvard University Archives. 
Parsons, Talcott. 1977. Social Systems and the Evolution of Action Theory. New York: The Free Press.

Preglau, Max. Soziale Kausalität. Email an Helmut Staubmann vom 11.3.2016

Schluchter, Wolfgang (hrsg.) 1979. Verhalten, Handeln und System. Talcott Parsons ‘ Beitrag zur Entwicklung der Sozialwissenschaften. Frankfurt am Main: Suhrkamp.

Stammer, Otto (hrsg.) 1965. Max Weber und die Soziologie heute: Verhandlungen des 15. Deutschen Soziologentages [vom 28. bis 30. April 1964 in Heidelberg; aus Anlaß des 100. Geburtstages Max Webers]. Tübingen: Mohr.

Staubmann, Helmut. 2015. Marx, Parsons, and the Quicksands of History. Harold J. Bershady's Intellectual and Personal Life Journey. European Journal of Cultural and Political Sociology 2: 56-61

Staubmann, Helmut. 2016. Talcott Parsons und die Kultursoziologie. In: Handbuch Kultursoziologie. Band 1: Begriffe-Kontexte-Perspektiven-Autor_innen, hrsg. Stephan Moebius, Frithjof Nungesser und Katharina Scherke. Wiesbaden: Springer.

Staubmann, Helmut, Tschernitz, Maria. 2016. Rezeption des amerikanischen Strukturfunktionalismus in der deutschsprachigen Soziologie. In: Handbuch Geschichte der deutschsprachigen Soziologie. Band 1: Geschichte der Soziologie im deutschsprachigen Raum, hrsg. Stephan Moebius und Andrea Ploder. Wiesbaden: Springer.

Helmut Staubmann, Univ. Prof. für Soziologische Theorie und Kultursoziologie und Dekan der Fakultät für Politikwissenschaft und Soziologie an der Universität Innsbruck. 2013-15 war er Präsident der Österreichischen Gesellschaft für Soziologie.

Seine Publikationen im Bereich Kultursoziologie beschäftigen sich vor allem mit Fragen soziologischer Ästhetik (Die Kommunikation von Gefühlen, Duncker \& Humblot 1995; Hg. mit Alan Scott: Georg Simmel: Rembrandt: An Essay in the Philosophy of Art, Routledge 2005; Ästhetik - Aisthetik - Emotionen. Soziologische Essays, UVK 2008; Hg.: The Rolling Stones. Sociological Perspectives, Lexington 2013). Mit seiner Theoriearbeit steht er in der Tradition der soziologischen Systemtheorie. Aus dem aktuellen Projekt „The Unpublished Legacy of Talcott Parsons“ (gem. mit Victor M. Lidz) entstanden die Editionen Talcott Parsons and Winston White: Values of American Society (LIT 2016) und Subsystems of American Society (LIT 2017), sowie der Band Rationality in the Social Sciences. The Schumpeter/Parsons Seminar 1939/40 and Current Perspectives (Springer 2017). Mit Victor M. Lidz gibt er die Schriftenreihe Studies in the Theorie of Action (LIT) heraus. 